\title{
On Plant Modularity Traits: Functions and Challenges
}

\author{
Gianluigi Ottaviani, ${ }^{1, *}$ Jana Martínková, ${ }^{1}$ Tomáš Herben, ${ }^{2,3}$ \\ Juli G. Pausas, ${ }^{4}$ and Jitka Klimešová ${ }^{1}$

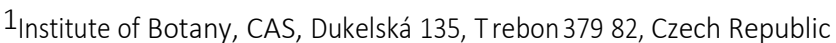 \\ 2 Institute of Botany, CAS, Zámek 1, Pru honice 252 43, Czech Republic \\ 3 Department of Botany, Faculty of Science, Charles University, Benátská 2, Praha 128 44, Czech Republic \\ ${ }^{4}$ CIDE-CSIC, C. Naquera Km 4.5, Montcada, Valencia 46113, Spain \\ *Correspondence: gianluigi.ottaviani@ibot.cas.cz (G. Ottaviani)
}

On-spot persistence, space occupancy, and recovery after damage are key plant functions largely understudied. Traits relevant to these functions are difficult to assess because of their relationships to plant modularity. We suggest that developing collection protocols for these traits is feasible and could facilitate their inclusion in global syntheses.

Plant Modularity Traits and Related Functions

After several decades of data collection, broad-scale analyses of plant functional traits (e.g., specificleaf area, plant height, seed size) are emerging as formidable tools for understanding plant ecological strategies [1-3]. Coordination and trade- offs among traits prevail, with a large proportion of the intra- and interspecific trait variability explained by two dimensions [2]: competitive ability and resource acquisition, retention and use strategies (see Glossary). Other important functions and associated ecological strategies, increases plant longevity and fitness [5]. Also, damaged plants mayregenerate not only from seed banks but also from bud banks [6]. Plant size and competitive ability may be related not only to vertical growth (as in trees) but also to horizontal growth, as in clonal plants. By means of spacers such as rhizomes and stolons, clonal plants can explore and forage for resources far from the maternal plant [7].

There are other key functions related to plant-plant interactions and plant-environment relationships that have long been considered essential for plant community ecology, yet they are unexplored. These include onspot persistence, space occupancy, and recovery after damage [4]. Traits related to these functions - plant modularity traits (Figure 1 and Box 1) - are underrepresented in global trait databases, with a few exceptions from Central Europe (e.g., CLO-PLA, LEDA; with information on plant clonality) and from fireprone regions (e.g., BROT; with information on resprouting). This gap has precluded global comparisons and is likely to reflect the lack of standardized protocols for measuring plant modularity traits [8]. The vast diversity and variability of clonal growth forms, the difficulty in identifying and locating the different modules, and their plasticity have limited the incorporation of modularity traits into plant functionalhandbooks.

We propose that such limitations can now be overcome by changing the way we look at individual plants; instead oflooking at organs like leaf, seed, and root, we should consider plant modules (Box 1). With this view we can understand how individual modules (e.g., shoots) are added or replaced, how long they persist, where they are located, and whether they are capable of becoming independent. With this 'modular perspective', we suggest operational protocols for describing traits relevant to the key functions of on-spot persistence, space occupancy, and recovery after damage. 
is, the modules for which traits must beassessed(Box 1 ). Relevant modules may differ among growth forms. Therefore, the correct determination of modules for the standardization of these traits is crucial. Below, we identify methods and challenges for the collection and standardization of key modularity traits related to: (i) longevity (on-spot persistence); (ii) clonality (space occupancy); and (iii) resprouting (recovery after damage) (Figure 1). Wealso include examples of successful attempts to dealing with these functions.

\section{Longevity}

Longevityreferstothelifespan of different plant modules and provides information on the time interval during which a plant occupies an area, acquiring, retaining, and sharing resources; itisthus indicative of overall plant persistence. Several longevity traits can be described using anatomical and morphological methods for dating modules (herbchronology, dendrochronology) and for detecting connections among clonal offspring (Figure 1). By considering the longevity of structures other than leaves, we can improve our understanding of plant strategies (e.g., how longevity correlates among different modules and differs between modules and organs [9]?). Determining the age of clonal plants is challenging when the oldest parts are decaying or when the growth rings are obscured. Age may be estimated, how- ever, by indirect approaches based on demographical techniques [10].

Clonality

Clonality is the capacity to produce physically independent ramets, which promotes vegetative reproduction when conditions hinder seed production or seedling establishment. The ability to spread laterally by means of spacers enables plants to explore and colonize new environments, compete with neighbors, and forage for nutrients [7]. Additionally, the ability of ramets connected by rhizomes to share resources in heterogeneous environments may be beneficial under stressful conditions. Key traits describing clonality include the type of clonal growth organs, the lateral spread, and the multiplication rate (Figure 1). Standardizing the descriptions of clonal growth organs is difficult because of the different morphological typologies used (e.g. [11]). Furthermore, clonality exhibits both high intraspecific plasticity and inter- specific variability, with many species combining several modes of clonal growth. Examples of successful imple- mentation of a common, shared typology for clonal organ description across bio- mes are still lacking, whereas definitions of individual clonal traits exist regionally and may serve as a foundation for broaderstandardization[11].

\section{Resprouting}

Resprouting - the ability to generate new shoots after the elimination of part or all of the aboveground biomass - is probably the most-studied strategy related to plant modularity worldwide, due to its relevance in fire-prone environments $[6,12]$. Resprouting allows plants to recover after seasonal rest or after injury caused by environmental pres- sures or disturbances. Most studies have recorded resprouting ability as a binary trait (presence/absence), but there is much more variability in the probability and the vigor of resprouting at the global scale [12]. Resprouting depends on two resources, buds and carbohydrates $[6,7,12]$, and their numbers and quanti- ties can be used as proxies for resprout- ing potential. Traits deserving focus include bud bank size, distribution of buds in relation to the soil surface, bud protection by plant structures (e.g., bark), and carbohydrate amount and concentration in roots and in specialized storage organs (Figure 1). The main chal- lenge in sampling these traits is the belowground location of most budbearing organs, where they are hard to extract and often intermingled among plants, making any quantitative determination rather difficult. Another challenge is standardizing the resprouting ability or vigor across different disturbance regimes. The knowledge gained in fire- prone ecosystems $[6,12]$ might serve as a basis for the development of standardization protocols.

\section{Concluding Remarks}

We suggest that the gathering of com- parable data on plant modularity traits is now an important and reachable target in plant ecology. By building on the above- mentioned expertise gained regionally regarding longevity, clonality, and resprouting traits, we are aiming at developing a large research network that would allow us to combine this knowl- edge into a handbook of standardized protocols. These procedures could then be applicable worldwide, considering these traits from different regions and biomes, which will increase our mechanistic understanding of plant fitness, strategies, and community assembly. Therefore, standardized methodologies could facilitate the inclusion of key, yet poorly studied, ecological dimensions into global syntheses. 
The research was supported by the Czech Science Foundation (Centre of Excellence PLADIAS, 1436079G and GA16-19245S). The authors thank the anonymous reviewers and the editor for challenging their ideas, which has strengthened the scientific message.

\section{References}

1. Wright, I.J. et al. (2004) The worldwide leaf economics spectrum. Nature 428, 821-827

2. Díaz, S. et al. (2016) The global spectrum of plant form and function. Nature 529, 167-171

3. Grime, J.P. (2001) Plant Strategies, Vegetation Processes and Ecosystem Properties. (2nd edn), Wiley

4. Weiher, E. etal. (1999) Challenging Theophrastus: a common core list of plant traits for functional ecology. J. Veg. Sci. 10, 609-620

5. Aarssen, L.W. (2008) Death without sex - the 'problem of the small' and selection for reproductive economy in flowering plants. Evol. Ecol. 22, 279-298

6. Pausas, J.G. and Keeley, J.E. (2014) Evolutionary ecology of resprouting and seeding in fire-prone ecosystems. New Phytol. 204, 55-65

7. Klimešová, J. et al. (2016) Herbs are different: clonal and bud bank traits can matter more than leafheight-seed traits. New Phytol. 210, 13-17

8. Pérez-Harguindeguy, N. et al. (2013) New handbook for standardised measurement of plant functional traits world- wide. Aust. J. Bot. 61, 167-234

9. Nobis, M.P. and Schweingruber, F.H. (2013) Adult age of vascular plant species along an elevational land-use and climate gradient. Ecography 36, 1076-1085

10. de Witte, L.C. and Stöcklin, J. (2010) Longevity of clonal plants: why it matters and how to measure it. Ann. Bot. 106, 859-870

11. Klimešová, J. et al. (2017) CLO-PLA: a database of clonal and bud-bank traits of the Central European flora. Ecology 98, 1179

12. Pausas, J.G. et al. (2016) Towards understanding resprouting at the global scale. New Phytol. 209, 945-954

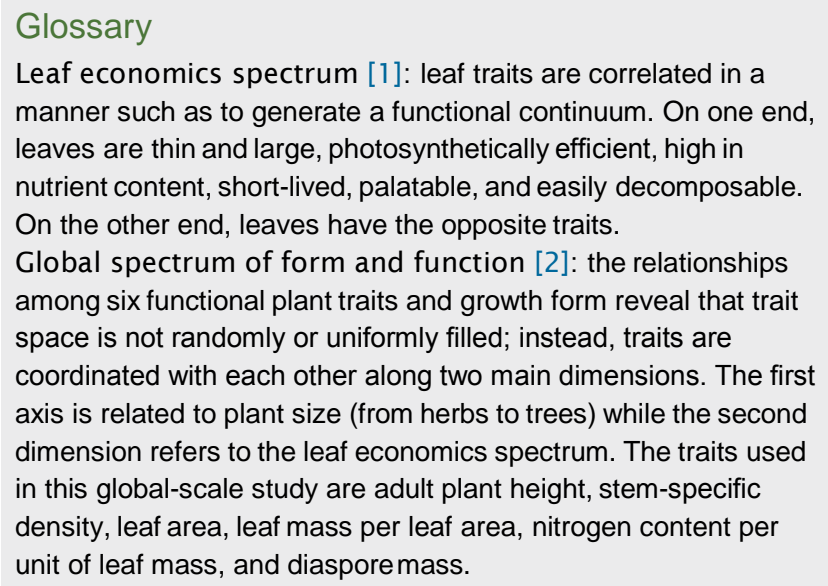




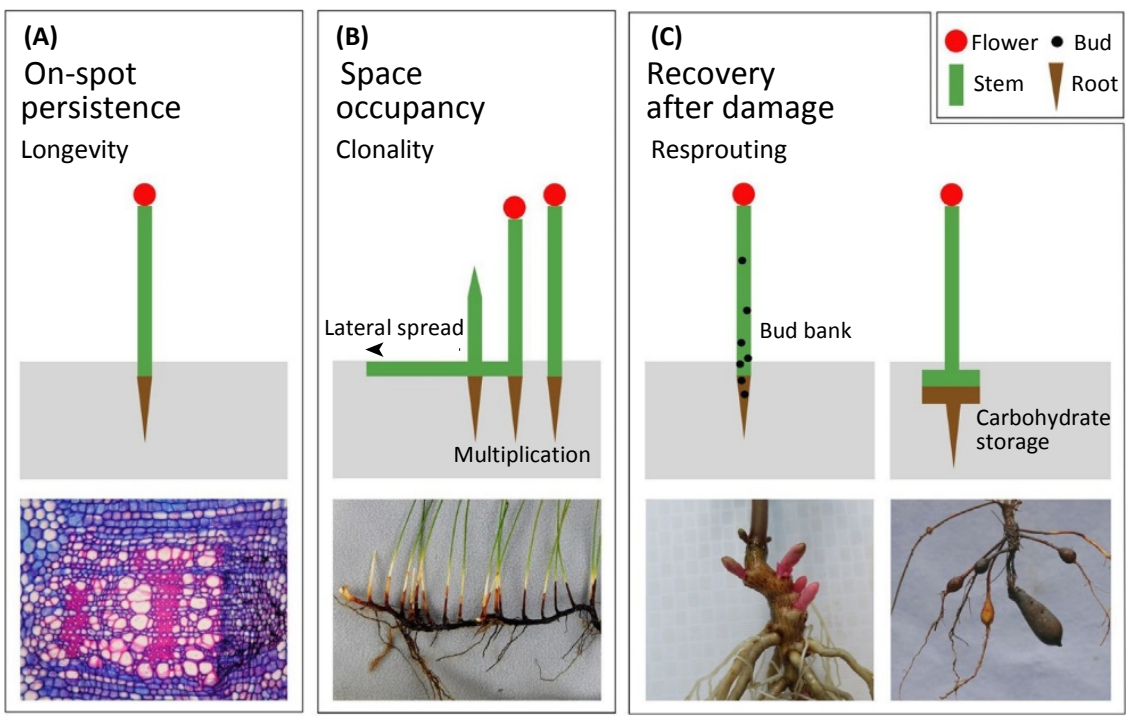

Figure 1. Plant Ecological Functions and Relevant Modularity Traits. (A) On-spot persistence is associated with plant longevity. Photograph: Transverse section of 3-year-old rhizome segment of Filipendula ulmaria (Rosaceae), 200x magnification (photograph credit: F.H. Schweingruber). (B) Space occupancy can be explored by clonality, which involves lateral spread, clonal growth organs, and multiplication rate. Photograph: Rhizome of Ficinia nodosa (Cyperaceae) with multiple shoots and roots (photograph credit: J. Martínková). (C) Recovery after damage depends on resprouting ability, which is made possible by two plant resources: reserves of buds and storage of carbohydrates. Left photograph: Root buds induced by injury in Euphorbia illirica (Euphorbiaceae) (photograph credit: J. Martínková). Right photograph: Storage root tubers in Filipendula vulgaris (Rosaceae) (photograph credit: Š. Jane®ek).

\section{Box 1. Plant Modularity: Basic Concepts}

Plantmodules are defined as any repeating architectural unit of a plantbody. Clonal and non-clonal plants can be formed by differentmodules (Figure I), determining their architecture and associated functions. Four major modules are identified.

- Genet: Product of a zygote (the rooting unit in non-clonal plants).

- Ramet: Potentially independent part of a genet (a rooting unit in clonal plants).

- Rooting unit: The smallest plant part capable of independently surviving as the root and shoot systems are connected (i.e., a ramet of clonal plants, the genet of non-clonal plants).

- Shoot: The product of apical meristems; its growth is usually terminated by flowering and fruiting structures. Examples are ramets in clonal herbs and twigs in trees.

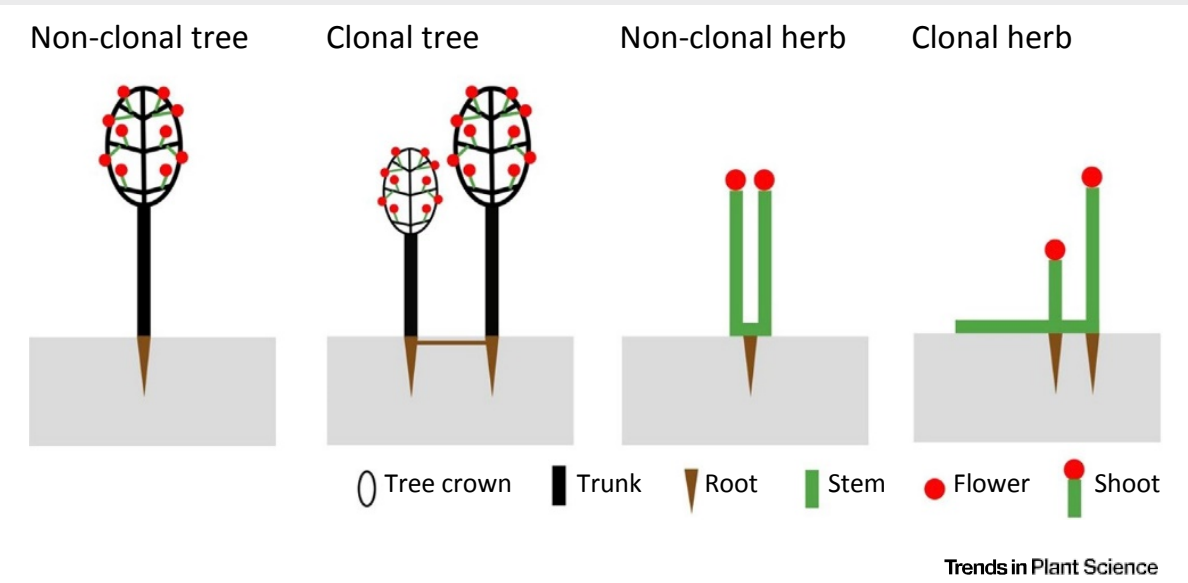

Figure I. Modular Structures in Non-clonal and Clonal Trees and in Non-clonal and Clonal Herbs. Inthese examples, the non-clonal tree and non-clonal herb are formed by one rooting unit (i.e., the rooting unit is the genet) but their clonal counterparts are formed by two rooting units (the rooting unit is a ramet). 